\title{
Performance of MIMO-Based Wireless Sensor Networks with Cochannel Interference
}

\author{
\#A.M. Abbosh and D.Thiel, senior member IEEE \\ Center for Wireless Monitoring and Applications, Griffith University, Nathan Qld, Australia 4111. \\ abbosh@itee.uq.edu.au
}

\begin{abstract}
Energy requirements of MIMO-based limited-energy wireless sensor networks are analyzed and simulated in the presence of the worst case cochannel interference and under different propagation conditions. The analysis and simulations were compared between three different types of systems, SISO, $M I M O$ and MIMO with CSI. Results demonstrate the robustness of MIMO based wireless sensor networks in spite of the strong cochannel interference that may exist.

Index Terms- MIMO, SISO, Wireless Sensor Network.
\end{abstract}

\section{INTRODUCTION}

Wireless sensor networks (WSN) are usually composed of many wireless nodes that are distributed in a certain fashion to cover the area to be monitored. Those nodes typically operate with small batteries for which replacement, if possible, is very expensive and difficult. Thus, the wireless nodes must operate without battery replacement for as long as possible. Consequently minimizing the energy consumption is vital when designing those systems.

Multiple-input multiple-output (MIMO) have been proposed for wireless sensor networks as it has been proven that MIMO-based systems can give the same throughput requirements with less transmission energy needed compared with single-input single-output (SISO) systems [1]. It was expected that due to the complex electronic circuitry needed for MIMO systems the total energy needed by those systems may exceed energy requirements of SISO systems. Therefore, several techniques were proposed in order to enhance performance of the wireless sensor MIMO-based systems [14]. The analysis made in those papers assumed a system with no cochannel interference.

Due to the wide spread of the use of WSN in particular and the personnel wireless systems in general and due to the limited frequency bands available then the effect of cochannel interference must be considered. In this paper the effect of cochannel interference on the performance of WSN is analyzed and simulated. Distribution of the systems in this paper is assumed to be for the hexagonal 7-cell reuse system. The systems are assumed to work with a full load, i.e. the worst case cochannel interference is assumed. Two types of propagation conditions are considered in simulations. (a) a "soft" propagation condition, in which the path loss exponent is 2 and (b) a "severe" propagation condition, in which the path loss exponent is 3.5 . The channel was modeled assuming clustered multipath incidence [5] and the fading distribution was assumed to be Rician in order to take effect of the lineof-sight rays into consideration. Two types of MIMO systems were investigated, one without channel state information (CSI), i.e. the transmitter has no information about the channel, and the other with CSI. Performance of those systems is then compared with that of SISO systems under the same conditions.

\section{MoDELS}

Consider a MIMO channel with $M$ antennas at the transmitter, $N$ antennas at the receiver and $K$ cochannel interfering signals each equipped also with $M$ transmitting antennas. The received signal at the desired receiver can be modeled as [6];

$y=H_{d} x_{d}+\sum_{i=1}^{K} \sqrt{P_{i}} H_{i} x_{i}+n$

where $H_{d}$ is $N \times M$ normalized channel matrix for the desired user, $x_{d}$ is $M \times 1$ transmitted signal vector for the desired user. Similarly, $P_{i}$ is the average access path loss of the $i^{\text {th }}$ interferer signal compared to that of the desired signal, $H_{i}$ is $N \times M$ normalized channel matrix of the $i^{t h}$ interferer and $x_{i}$ is $M \times 1$ transmitting signal vector of the $i^{\text {th }}$ interferer. Also $n$ is the additive complex Gaussian noise vector with zero mean and covariance value $\left(N_{o}\right)$.

The channel matrices $H_{d}$ and $H_{i}$ are formed using the method discussed in $[4,5]$. They include the effect of the Rician fading, with a Rice factor $=10 \mathrm{~dB}$. This means a strong line-of-sight ray is present and transmitter and receiver are correlated as well as the effect of mutual coupling between elements of the transmitting and receiving arrays. When forming the channel matrices, clustered multipath propagation 
was assumed. The number of clusters, power distribution within each cluster, angles-of-arrival and angles-of-departure are as in model $\mathrm{F}$-IEEE-802 [5].

From (1) the mutual information can be calculated using the following equation $[6]$;

$$
I=\log _{2}\left(\operatorname{det}\left(I_{N}+H_{d} R_{d} H_{d}{ }^{H} B_{I}{ }^{-1}\right)\right)
$$

where

$$
B_{I}=H_{I} R_{I} P_{I} H_{I}{ }^{H}+N_{o} I_{N}
$$

and

$$
R_{d}=E\left(x_{d} x_{d}{ }^{H}\right)
$$

where $I_{N}$ is $N \times N$ identity matrix, $H_{I}$ is $N \times N_{I}$ matrix which is result of composition of the interferers matrices $H_{i}$ where the first $M$ columns of $H_{I}$ comes from matrix of the first interferers and the second $M$ columns are channel matrix of the second interferer and so on $N_{I}$ is the total number of interfering antennas and it is equal to $\left(K^{*} M\right)$. The $N_{I} \times N_{I}$ diagonal matrix $P_{I}$ is formed from $P_{i}$ where the first $M$ elements of the diagonal comes from effect of the first interferer and so on. The $N_{I} \times N_{I}$ diagonal matrix $R_{I}$ is formed by the same way from the elements;

$$
R_{i}=E\left(x_{i} x_{i}^{H}\right)
$$

The superscript $(H)$ in all equations indicates the Hermitian operation.

In this paper we assumed many WSN are working nearby to the system under consideration. Distribution of the systems and frequency allocation were chosen according to the 7-cell reuse system, although any other configuration could also be assumed. Therefore, under full load conditions the desired user will suffer from six cochannel interferers [7], i.e;

$$
K=6
$$

The following scenarios were considered in this paper. The first one is MIMO without CSI and the power is assumed to be equally divided between elements of the array. If we assume that all sensors transmit the same power then;

$$
R_{d}=R_{1}=R_{2}=\ldots=R_{N I}=\frac{\bar{E}_{b}}{M}
$$

where $\bar{E}_{b}$ is the total energy per bit transmitted from each sensor. In order to calculate the access path loss $P_{i}$ and hence $P_{I}$ the two-slope path loss model is considered [5];

$$
L_{p}=\left\{\begin{array}{ll}
\left(\frac{4 \pi d}{\lambda}\right)^{2} & \text { for } d<d_{b r} \\
\left(\frac{4 \pi d_{b r}}{\lambda}\right)^{2}\left(\frac{d}{d_{b r}}\right)^{n} & \text { for } d>d_{b r}
\end{array}\right\}
$$

where $L_{p}$ is the path loss, i.e. the ratio of transmitted power to received power, $d$ is the transmitter-receiver separation, $\lambda$ is the wavelength, $n$ is the path-loss exponent after the break-point distance and $d_{b r}$ is the break-point distance which depends on wavelength and heights of transmitter and receiver antennas. In this paper we assumed $d_{b r}=10 \mathrm{~m}$. The position of the desired user is assumed to be random within its cell. Its position relative to the center of the cell is $r_{d}$ where $0 \leq r_{d} \leq R$ assuming that the cell has a radius equal to $R$. The distance between the center of the desired cell and the center of any of the interfering cells is $D$ and its value can be calculated from [7];

$$
D=\sqrt{21} R
$$

We assume that the $i^{\text {th }}$ cochannel sensor is at a distance $r_{i}$ from the center of its cell. The desired sensor and the $i^{t h}$ interferer are assumed to be at angles $\theta_{d}$ and $\theta_{i}$ respectively with respect to $D$ (see Fig.1). The distance $d_{i}$ between that interferer and the desired sensor can be calculated geometrically from Fig.1. The parameters $r_{d}$ and $r_{i}$ are assumed to take any value between 0 and $R$ with uniform distribution while the parameters $\theta_{d}$ and $\theta_{i}$ can take any value between 0 and $360^{\circ}$ with uniform distribution. If the client of the WMS under investigation is in the center of the cell then the path loss between the client and the desired sensor $\left(L_{p d}\right)$ can be calculated from (8) by substituting $d$ with $r_{d}$ while the cochannel interferer path loss, $L_{p i}$ is calculated by replacing $d$ with $d_{i}$. The average access path loss for the $i^{t h}$ interferer can now be found as $\left(L_{p d} / L_{p i}\right)$, therefore; 


$$
P_{i}=\left(L_{p d} / L_{p i}\right)
$$

In this equation we assumed the same average path loss between any antenna element in transmitter of the interferer and any antenna element in the desired receiver. This assumption comes from the fact that the effect of fast fading was included in the channel matrix. Also, the path losses in (10) are calculated and averaged over 1000 randomly selected positions for the desired sensor and the interferers.

The signal to interference and noise ratio (SINR) can be calculated from (2) by summing all the positive eigen values of the second factor in (2);

$$
\operatorname{SINR}_{M I M O}=\sum \operatorname{eig}\left(\frac{\bar{E}_{b}}{M} H_{d} H_{d}{ }^{H} B_{I}{ }^{-1}\right)
$$

Substituting for $R_{i}$ from (7) into (3) and then (11);

$$
\operatorname{SINR} R_{M M O}=\sum \operatorname{eig}\left(H_{d} H_{d}^{H}\left(H_{I} P_{I} H_{I}^{H}+M N_{o} / \bar{E}_{b}\right)^{-1}\right)
$$

The second scenario is MIMO+CSI. In this case the waterfilling algorithm was used, i.e. non-uniform distribution of power between array elements. The main issue in WSN is how to minimize the energy consumption. To achieve that goal it is important to decrease the electronic circuitry required and decrease the transmission energy. In [4] it has been shown that it is better to use CSI in WSN to direct transmission and reception using the best channel and to use other channels just to monitor variation in channel condition. Hence, SINR in this case is equal to the maximum value of the eigen values of (11) after assuming that the total power will be transmitted and received using a single channel ( the best channel);

$$
\operatorname{SINR}_{M I M O+C S I}=\max \operatorname{eig}\left(\bar{E}_{b} H_{d} H_{d}{ }^{H} B_{I}{ }^{-1}\right)
$$

The cochannel interferers may also use the principle of best channel transmission. In that case each cochannel sensor will choose the best channel with respect to its client. This means that the average cochannel effect will be decreased compared to the equal power distribution among all channels. If we still assume the cochannel effects to be equivalent to the previous case then;

$$
\operatorname{SINR}_{M I M O+C S I}=M^{*} \max \operatorname{eig}\left(H_{d} H_{d}{ }^{H} A^{-1}\right)
$$

where;

$$
A=H_{I} P_{I} H_{I}{ }^{H}+M N_{o} / \bar{E}_{b}
$$

Comparing $(14,15)$ with $(12)$ it is clear that SINR in case of MIMO+CSI is greater than its value with MIMO. The two scenarios mentioned above will be compared to the SISO model. SINR for the SISO model can be considered as a special case of the MIMO model with $N=M=1$.

\section{Energy Calculation}

In order to compare the performances of different systems, the total energy requirements of those systems should be considered. Besides the transmission energy, the power consumption of the electronic circuits in the transmitting and receiving nodes must be included. The electronic circuits used in the transmitter and receiver are assumed to be as in Fig.2 for the SISO system. For the MIMO system there will be $N$ parallel branches of the receiver circuits and $M$ branches of the transmitter circuits that are shown in Fig.2. The power requirements for different parts of the electronic circuitry of SISO or MIMO system are assumed to be as in [1].

The following equations are used to estimate the energy requirements $[1,3]$.

$$
\begin{aligned}
& E_{b}=(1+\alpha)\left[\bar{E}_{b} * \frac{M a r^{*} N_{f}}{G_{t} G_{r}} * L_{p}\right]+\frac{P_{c}}{R_{b}} \\
& P_{c}=M P_{t}+2 P_{s}+N P_{r} \\
& B_{e}=\operatorname{Mean}[Q(\sqrt{2 * S I N R})]
\end{aligned}
$$

where $E_{b}$ is the total energy consumption per bit. This includes $\bar{E}_{b}$ as well as the energy consumed by the electronics, $\alpha$ defines the amplification characteristics of the power amplifier at the transmitter, Mar is the safety margin for the system, $N_{f}$ is noise figure of the receiver, $G_{t}$ and $G_{r}$ are the gains of the antenna elements at the transmitter and receiver side respectively, and $R_{b}$ is the bit rate. $P_{c}, P_{t}, P_{s}$ and $P_{r}$ are the power consumption values of the circuit sections for one branch of; the transmitter digital-toanalog converter, mixer and filter; the synthesizer; and the receiver low-noise amplifier, mixer, intermediate frequency amplifier, filter and analog-to-digital converter. The $\mathrm{Q}$ function in (16c) is the Gaussian error integral and $B_{e}$ is the bit error rate. The word (Mean) in (16c) indicates that the estimation should be averaged over all the channel matrix possibilities.

It is important to remember that in the case of MIMO+CSI there must be $M$ branches of transmitter circuitry and $N$ branches of the receiving circuitry. While the signal is transmitted and/or received by one branch, the additional ( $M$ - 
1) and $(N-1)$ branches are needed to estimate the channel matrix and hence update the information about the best channel pipe. The additional $(M-1)$ and $(N-1)$ branches are only activated at time intervals which must be comparable to the channel coherence time $\left(T_{c}\right)$, say for $\beta T_{c}$ where $0<\beta \leq 1$. This is because when the time interval is less than $T_{c}$, the channel will be stable and time-invariant but after that, the channel may change. Therefore, when using MIMO+CSI, (16b) becomes;

$$
P_{c}=P_{t}+2 P_{s}+P_{r}+\left[(M-1) P_{t}+(N-1) P_{r}\right] /\left(\beta T_{c} R_{b}\right)
$$

In the simulations that follow, it is assumed that, $\beta=0.5$ and $T_{c}=2 m s$.

\section{RESUlts AND Discussions}

In these simulations it was assumed that the radius of the area to be covered by any WSN, i.e. radius of the cell $R$, is $100 \mathrm{~m}$. The path-loss exponent $n$ for the interfering signals is considered to be 3.5 while two cases were considered for the path-loss exponent of the desired signal. The first case is $n=2$ where almost free space propagation is assumed and the second case, $n=3.5$ when difficult propagation conditions exist due to un-avoidable obstacles. In the simulations the frequency of operation is assumed to be $2.5 \mathrm{GHz}$ and the bit rate is $10 \mathrm{kbps}$ while the probability of error is 0.001 .

Variation of the total energy required per bit $\left(E_{b}\right)$ for MIMO, MIMO+CSI and SISO wireless sensor networks with distance is shown in Fig.3. It is obvious from Fig.3a that when the desired signal propagates in a near free-space propagation while the cochannel interferer suffers from severe propagation conditions, cochannel interference has almost no effect on performance of MIMO systems (less than $1 \%$ increase in energy at the edge of the cell) while it has a great impact on SISO system (about $80 \%$ increase in energy at edge of the cell) although SISO still needs the lowest energy within a short distance, i.e. up to a distance of about $15 \mathrm{~m}$ in these simulations. When the propagation conditions become severe for the desired signal, then the effect of the cochannel is great on SISO. This requires too much energy to maintain the desired probability of error. For example at the edge of the cell, the required percentage increase in energy to maintain the planned probability of error is 6000 (see Fig.3b).

For MIMO systems the effect is relatively small. For example at the edge of the cell, the required energy increases by $25 \%$ for MIMO and $18 \%$ for MIMO+CSI compared with the noise limited case. Finally it is important to note that although SISO appears to do well in short distances, several techniques have been proposed to enhance the performance of MIMO systems that lower the energy requirements, less than that of SISO systems even for very short distances [1-4].

\section{Conculsion}

Simulations in this paper show that MIMO systems are robust in the presence of cochannel interference when used in wireless sensor networks. This will strengthen their position against SISO systems in applications that could suffer from a high level of cochannel interference. As frequency bands are a limited natural resource, the huge increase in wireless systems will increase the effect of cochannel interference. In that case, systems that can minimize or even cancel these effects will have a bright future.

\section{ACKNOWLEDGMENT}

The authors acknowledge the financial support of DESTAustralia via AEI-International Education Network.

\section{REFERENCES}

[1] S. Cui, A. Goldsmith and A. Bahai, "Energy-efficiency of MIMO and cooperative MIMO Techniques in sensor networks", IEEE Journal on Selected Areas in Comm., vol.22, no.6, pp. 1089-1098, Aug. 2004.

[2] L. Xiao and M. Xiao, "A new energy-efficient MIMOsensor network architecture M-SENMA", IEEE Vehicular Tech. Conf., Vol.4, pp. 2941-2945, USA, Sept. 2004.

[3] S. Cui, A. Goldsmith and A. Bahai, "Energy-constrained modulation optimization", to appear in IEEE Trans. Wireless Communications, Sept 2005.

[4] A.Abbosh and D.Thiel, "Comparison between SISO, MIMO and SPA in limited-energy wireless systems" submitted to IEEE Trans. Wireless Communications, 2005.

[5] V. Erceg et. al, "TGn channel models", IEEE 802.11 document 03/940r4, May 2004.

[6] M.Kang, L. Yang and M. Alouini, "Certain computations involving complex Gaussian Matrices with applications to the performance analysis of MIMO systems", Chapter3 in Space-Time Processing for MIMO Communications, John Wiley \& Sons, Canada, 2005.

[7] B. Walke, Mobile radio networks; Networking, protocols and traffic performance, John Wiley \& Sons, 2nd Edition, New York, 2002. 


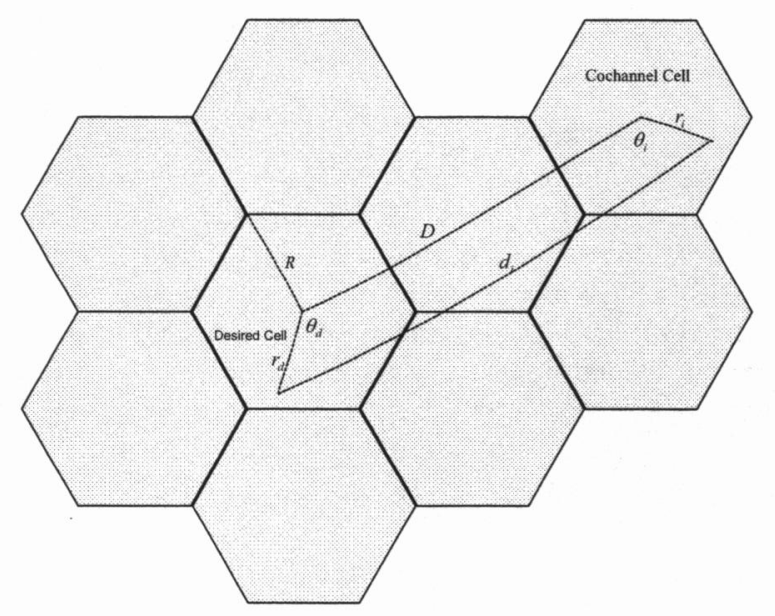

Fig.1: Schematic diagram showing position of the desired sensor and the cochannel interferer assuming a 7-cell reuse distribution.

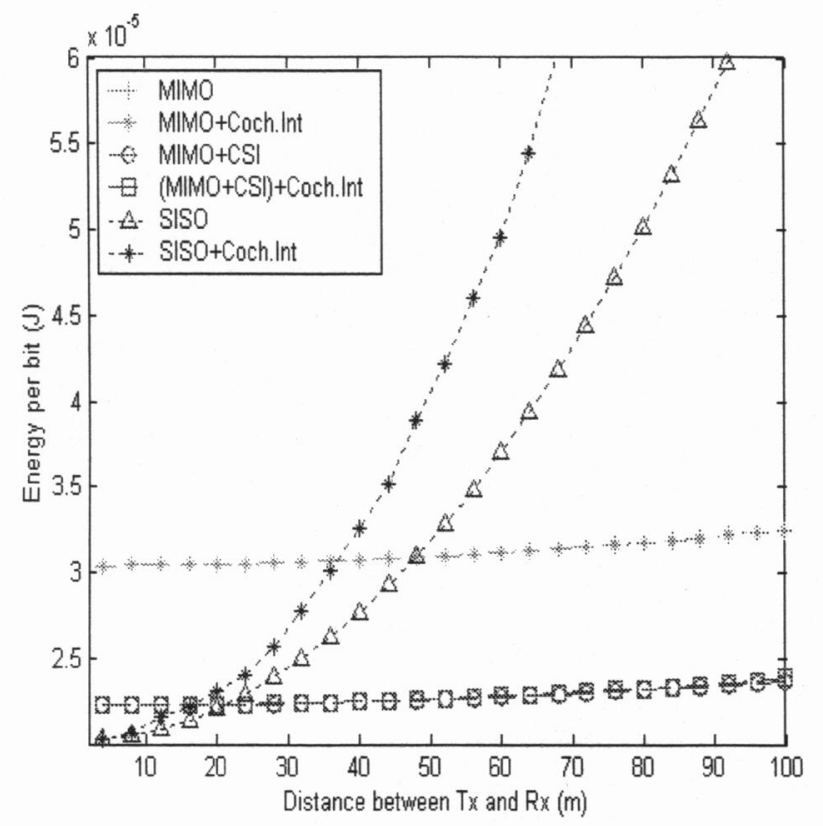

(a) $n=2$
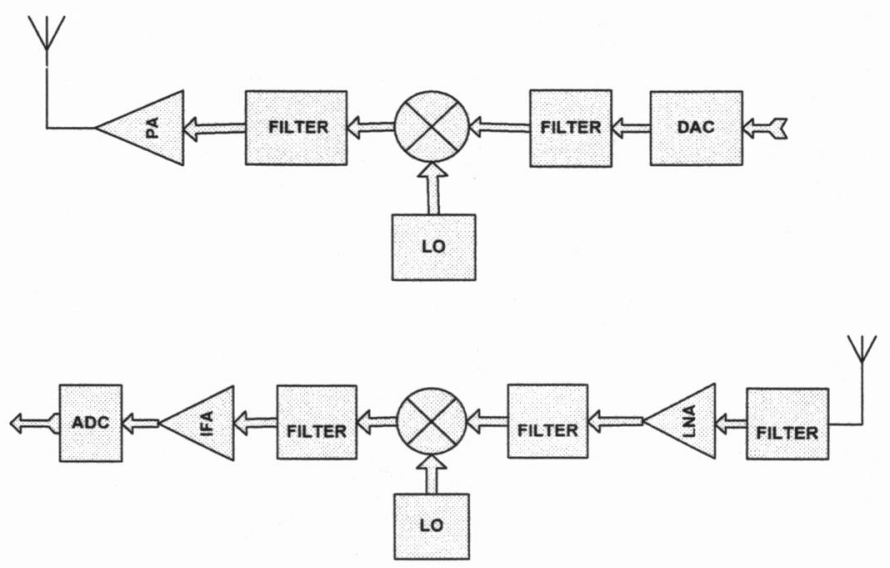

Fig.2: Block diagram of the transmitter (upper part) and the receiver (lower part).

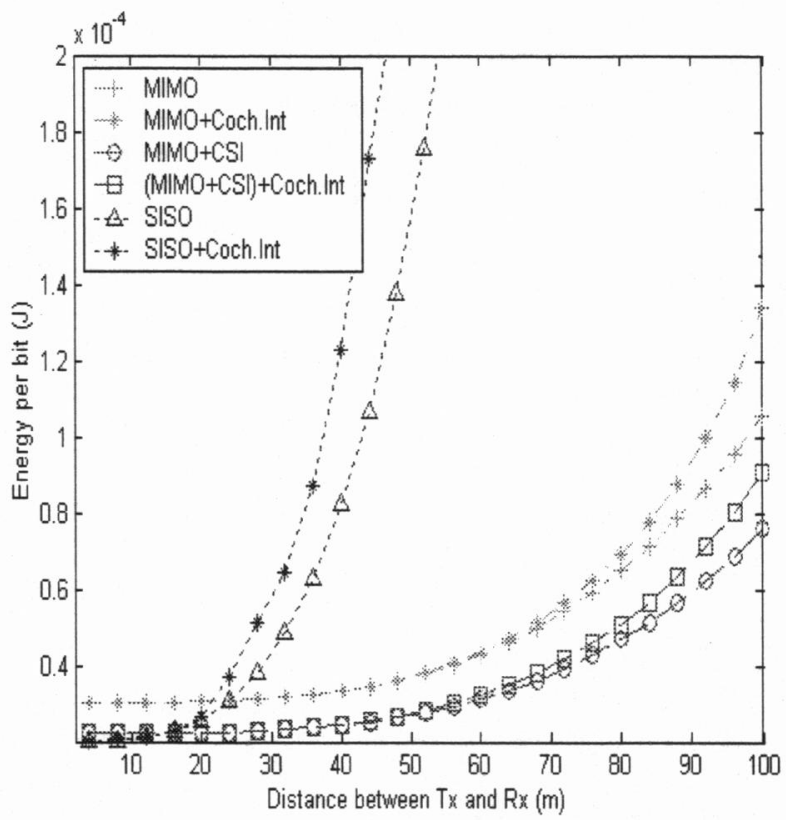

(b) $n=3.5$

Fig.3: Effect of cochannel interference on required energy per bit for wireless monitoring systems using MIMO, MIMO+CSI and SISO. 